\title{
Laparoscopic Sleeve Gastrectomy Complicated by Mesenteric Vein Thrombosis, Abdominal Compartment Syndrome and Pulmonary Emboli: Case Report
}

\author{
Erika Leung*, MD, MSc; G. Brent Sorensen, MD \\ University of Missouri-Kansas City, Kansas City, MO, USA \\ Saint Luke's Hospital of Kansas City, Kansas City, MO, USA
}

\begin{abstract}
Background: Obesity is a growing problem all over the world, including the United States. Single-incision laparoscopic sleeve gastrectomy is a surgery performed for patients who want to lose weight. The number of deaths resulting from thromboembolic complications from bariatric surgeries continues to be of major concern.

Case Description: A 38-year-old female was admitted for single incision sleeve gastrectomy and was discharged home three days later. Subsequently she began to have abdominal pain, nausea and vomiting. A CT scan revealed superior mesenteric vein thrombosis with small bowel ischemia, splenic infarction and main and right portal vein branch thrombosis. An exploratory laparotomy demonstrated necrotic bowel due to abdominal compartment syndrome, and an area of small bowel was resected due to internal hernia. Surgical management of the patient during her second hospital stay included a decompressive laparotomy, internal hernia reduction, a small bowel resection.

Discussion: Superior mesenteric vein thrombosis can be a life-threatening complication and present with non-specific presentations; thus, it is imperative that it is identified and managed promptly as these cases carry significant morbidity and mortality. Obese patients who undergo bariatric surgery frequently have other co-morbidities; many of which can complicate a case further. Mesenteric vein thrombosis is normally treated with unfractionated or low-molecular-weight heparin. (Int J Bopmed. 2015;5(3):162-166.)
\end{abstract}

Keywords: mesenteric vein thrombosis; laparoscopic sleeve gastrectomy; obesity; bariatric surgery.

\section{Introduction}

Obesity is a growing problem all over the world, including the United States. The prevalence of obesity continues to rise steadily over the last three decades and is likely to remain on the rise [1]. Bariatric surgery is the most effective treatment of morbid obesity and depending on the type of operation; this surgery can also be effective in resolving diabetes [2]. Single-incision laparoscopic sleeve gastrectomy (SILSG) is a surgical procedure for patients who want to lose weight. The operation involves a reduction in stomach size, and the patient is left with a stomach that resembles a tube, which can reduce the sensation of hunger because the part of the stomach that is removed would ordinarily secrete hunger hormones. While

*Corresponding author: Erika Leung, MD, MSc. St Luke's Hospital, Kansas City, MO,USA.Email: Erika.leung@mail.mcgill.ca the recovery period for such surgeries is often uneventful, one particular case was complicated by the development of a superior mesenteric vein thrombosis, abdominal compartment syndrome and pulmonary emboli. Superior mesenteric vein thrombosis can be a life-threatening complication and can present with non-specific presentations; thus, it is imperative that it is identified as soon as possible and treated immediately.

\section{Literature Search}

A PubMed search was performed using the keywords of "sleeve gastrectomy" and "thrombosis". Two other articles in the literature have reported cases similar to this one. "Superior Mesenteric Vein Thrombosis after Laparoscopic Sleeve Gastrectomy" by Pineda postulated that this was induced by a blunt and sharp dissection at the level of the distal stomach, which was close to the pylorus [3].

Bellanger et al. [4] presented three cases of mesenteric 
vein thrombosis development following laparoscopic sleeve gastrectomy. Their practice performed over 800 operations and had three cases of thromboses involving the superior mesenteric vein. Intravenous heparin was started and converted to oral warfarin on discharge for all three cases. No hypercoagulable disorder was discovered in any of the three cases [4].

In addition to the case studies above, D. Goitein et al. [5] performed a retrospective multicenter study and found $17(0.3 \%)$ patients out of 5706 who underwent laparoscopic bariatric surgery and had developed portomesenteric vein thrombosis. Patients were treated by anticoagulation with subcutaneous low-molecular-weight heparin $(n=15)$ or intravenous heparin $(\mathrm{n}=2)$, followed by warfarin sodium. Three patients were found to have genetic coagulation deficiencies. Management of such cases included rehydration and supportive care, anticoagulation, thrombolysis and if needed, laparoscopy. Acute postoperative portomesenteric vein thrombosis is a rare but life-threatening complication that can occur after SILSG. Early recognition is of paramount importance to prevent the progression into bowel infarction.

This case report is a discussion of the development of superior mesenteric vein thrombosis (SMVT), abdominal compartment syndrome and multiple pulmonary emboli in a patient who underwent SILSG. This location of venous thrombosis can be damaging to other organs and the hypoxic injury from abdominal compartment syndrome resulted in short bowel syndrome. Moreover, this patient developed pulmonary emboli during anticoagulant therapy while in hospital. These were rare occurrences but should be addressed as possible complications for this surgery.

\section{Case Description}

A 38-year-old African American female with a body mass index of $48.2 \mathrm{~kg} / \mathrm{m}^{2}$ was admitted for SILSG. The patient's initial physical examination, prior to sleeve gastrectomy, was unremarkable. She was then discharged. However, on readmission several days later, she appeared critically ill. Her vital signs were quite unstable. She was pyrexic and tachycardic with a blood pressure hovering around 100/63 $\mathrm{mmHg}$. She was saturating $98 \%$ to $100 \%$ on $3 \mathrm{~L}$ of oxygen on nasal cannula. Her abdomen was firm with diminished bowel sounds.

The patient's past medical history included obesity, hypothyroidism, hypertension and dyslipidemia. She was an ex-smoker having quit twenty years ago with one pack year history. Family history was negative.

The patient underwent SILSG and experienced some nausea and vomiting post-operatively, alleviated with antiemetics. She tolerated her bariatric full liquid diet and was discharged home three days later. Subsequently she began to have abdominal pain, nausea and vomiting. A CT scan revealed SMVT with diffuse small bowel ischemia, segmental splenic infarct, main and right portal vein branch thrombosis but no evidence of hepatic ischemia. A thrombectomy was performed by interventional radiology. An exploratory laparotomy demonstrated necrotic bowel due to abdominal compartment syndrome, and $208 \mathrm{~cm}$ of small bowel was resected; with a second reexploration and further resection two days later. She had a final abdominal closure whereby the wound was completely closed five days later. She had significant acidosis with $\mathrm{pH}$ of 7.19 , elevated lactate levels and she required intubation. She was on a heparin drip from the time of diagnosis and underwent bridging for warfarin. She then experienced sudden tachypnea, dyspnea and tachycardia and was found to have multiple pulmonary emboli with pulmonary infarcts on CT pulmonary angiogram. A followup CT scan of the abdomen at the end of her hospitalization showed extensive small bowel resection, extensive but stable SMVT, stable splenic infarction, and improved ascites. The patient eventually recovered from her illness and was discharged in a stable condition.

\section{Discussion}

SMVT can be a life-threatening complication and present with non-specific presentations; thus it is imperative that it is identified and managed promptly. The mortality rate of acute mesenteric thrombosis can range from $20 \%$ to $50 \%$ [6]. Presenting symptoms can be mild or severe, and rapid recognition is important. If treated early with anticoagulation and fluids, the progression of the disease can be limited. In addition to developing a mesenteric vein thrombosis, this patient subsequently developed abdominal compartment syndrome which led to short bowel syndrome and pulmonary emboli.

In recent years, laparoscopic sleeve gastrectomy (LSG) has been identified as a newer and innovative approach as a lone-standing surgery for morbid obesity. It is considered easier, faster, and less traumatic to perform for surgeons than laparoscopic Roux-en-Y gastric bypass (LRYGB) [7]. Bariatric surgery is the only evidence based treatment of morbid obesity with proven and sustained weight loss and improvement in comorbidities [8-10]. Initially, LSG was introduced as the first stage in a two-staged bariatric surgical approach but is now accepted as a stand-alone bariatric procedure. It is a desirable procedure comparatively because it reduces the gastric volume while preserving the continuity of the gastrointestinal tract [8]. The sleeve gastrectomy can preserve the integrity of the pylorus and does not include intestinal bypass while the Roux-en-Y does not [2]. In Li's review, the LRYGB group had a higher incidence of complications than the LSG group. The LSG procedure is also safer and less complex than LRYGB and it avoids the long-term sequela of micronutrient deficiency after duodenum exclusion that is seen in the Rouxen-Y procedure [2]. In general, bariatric surgery is successful in reducing the rate of diabetes; although the mechanism for type II diabetes mellitus remission is not particularly clear. V. Vage et al [8] found LSG to have acceptable morbidityrates and to be an effective procedure overall. It had high resolution rates for diabetes, hypertension, hyperlipidemia, sleep apnea, musculoskeletal pain, snoring, urinary leakage and amenorrhea [8]. There is also an association between LSG and GERD; in Vage's study, the patients who were treated for GERD symptoms preoperatively had resolution of their GERD 
symptoms postoperatively [8]. Other improvement shown was that ALT values could also significantly improve after the operation. Obesity associated with non-alcoholic fatty liver disease can also be resolved after bariatric surgery [11]. Some of the advantages include preservation of endoscopic access to the upper gastrointestinal tract, the lack of an intestinal anastomosis thus excluding the risk of internal herniation, normal intestinal absorption and prevention of dumping syndrome due to pylorus preservation [7]. B. Albeladi et al. [7] found that DMII was resolved in $100 \%$ of the patients in the LSG vs. $85.7 \%$ in the LRYGB group. However, in those where DMII was not resolved, the medication dosage was decreased. Hypertension was resolved in $53.8 \%$ of patients with LSG vs. $46.7 \%$ with the patients who underwent LRYGB [7]. Having some of these major comorbidities resolve is very favourable for LSG surgery. LSG is also less complex than LRYGB, and leads to a slower operative time as well [7].

Another type of surgery includes laparoscopic adjustable gastric banding surgery (LAGB). This surgery is simple and safe and restricts food by using an adjustable band by creating a pouch that is distended during meals. The banding surgery is similar to the sleeve gastrectomy because they both decrease the volume of the stomach to reduce food intake [12]. The advantages for LAGB include easier technique, shorter operative time, less invasive and fewer early complications. However, a meta-analysis comparing LAGB to LSG has shown that LSG had a better effect on morbid obesity in terms of weight loss and resolution of DMII [12]. Some of the common complications that come with gastric banding include migration of the band (slippage or displacement), band leak, esophageal spasms, and inflammation of the esophagus and GERD.

Reported complication rates for LSG are low, even though the patients undergoing the procedure are of high surgical risk. Major perioperative complications for LSG can include leaks and bleeding. In order to reduce bleeding risks, V. Vage et al. altered their regime for prophylaxis against thrombosis whereby prophylaxis is started post-operatively at a reduced dosage [8]. Some of the complications that can come with doing LSG include hemorrhage, staple line leak, abscess formation, and strictures. The source of the bleeding for hemorrhages can be intraluminal or extraluminal; symptoms include hematemesis or melena stools [13]. Staple line leaks are some of the more serious complications of LSG; and can occur in up to $5 \%$ of patients [14]. An early leak is considered one that is diagnosed within the first three days of surgery and a delayed leak is diagnosed more than eight days after surgery [15]. Treatment of the leak usually involves an abdominal washout with surgical repair. Intra-abdominal abscesses can also form as well, and this can be diagnosed with CT scans [13]. Stricture formation can also occur after LSG, presenting either acutely after surgery or later on. These are usually treated with conservative management; however if strictures become chronic, treatment can include endoscopic dilatation [13].

Because deficiencies in micronutrients in morbidly obese patients are frequent, a large number of patients already have nutritional deficiencies prior to bariatric surgery [16].
LSG is a restrictive procedure and lacks the malabsorptive component of LRYGB so the risk for developing deficiencies after surgery is usually considered low. Because of the resection of the fundus, some nutrients like iron or vitamin B12 are less likely to be absorbed [17]. Some patients also develop iron deficiency anemia and this can be explained by LSG. Iron needs to be transformed to an absorbable form by $\mathrm{HCl}$ in the normal stomach. The quantity of $\mathrm{HCl}$ produced in the stomach is reduced and nutrients can pass the stomach faster after LSG; thus this would be harder to absorb iron [18]. Folate deficiency is also common. Bone metabolism can also change after an LSG but this could also be due to weight loss. This can put them at risk for osteoporosis so this needs to be corrected or treated to avoid deficiency. It is important to supplement the patient after every bariatric procedure to avoid any nutritional deficiency [16]. The etiology for nutritional deficiencies after bariatric surgery is multifactorial but likely due to impaired absorption and decreased oral intake. The most common deficiencies include vitamin B12, vitamin D, folate, iron, and zinc. In general, micronutrient deficiencies were found to be less prevalent after sleeve gastrectomy compared to LRYGB [13]. Routine monitoring is warranted after the surgery so deficiencies can be diagnosed if necessary.

For this patient we chose to anticoagulate with enoxaparin. In a cohort study by Birkmeyer, they evaluated the effectiveness and safety of various different venous thromboembolism prophylaxis strategies for patients undergoing bariatric surgery. They had patients who used unfractionated heparin preoperatively and postoperative, patient who use unfractionated heparin preoperatively and low molecular weight heparin (LMWH) post operatively, and finally a third subset of patients who used low molecular weight heparin both preoperatively and postoperatively [19]. Rates for emboli development were 57\% lower for the unfractionated heparin/LMWH group and $66 \%$ lower for the LMWH/LMWH group when compared to the unfractionated heparin/unfractionated heparin group[19]. They concluded that low molecular weight heparin was more effective than unfractionated heparin for the prevention of venous thromboembolism and it did not increase the risk of bleeding in bariatric surgery patients. Another study has shown lower rates of bleeding with LMWH compared with unfractionated heparin in general surgery patients [20].

Similar to the othercases found in the literature, this patient was anticoagulated with heparin and bridged to warfarin. There are some suggested etiologies for the cause, including genetic deficiencies (Protein S, Protein C, Antithrombin III, and Factor V Leiden deficiency), prothrombotic states (sepsis, pregnancy, obesity, oral contraceptive use, cirrhosis, lupus anticoagulant, polycythemia vera, heparin-induced thrombocytopenia, and malignancy or myeloproliferative disorders), iatrogenic causes (trauma to the portal venous system, inflammation to the abdominal wall), neoplastic disorders or decreased portal flow $[5,6,21]$. Venous thromboembolism is a leading cause of mortality after bariatric surgery; the reported frequency of complications involving thromboembolic event in bariatric surgery patients ranges from $0.2 \%$ to $2.4 \%$. For this patient, the cause for the multiple thrombi was never discovered. 
Mesenteric thromboses have been described as either primary, where no cause is identified, or secondary where there are clear causes and predisposing factors; and 20 to $35 \%$ are primary cases [4]. Inappropriate anticoagulant dosing may lead to venous thromboembolism, particularly in obese patients. Frederiksen et al demonstrated that there was a negative correlation with body weight and the anticoagulant effect of a fixed dose of LMWH [22]. Given that the patient did not have a hypercoagulable disorder, it is difficult to determine the reason pulmonary emboli developed. However prolonged immobility, obesity, dehydration and recent surgery could all be seen as risk factors which put this patient in a hypercoagulable state.

The patient in this case study was obese, which increases the risk for hypercoagulability. Obesity is a pro-inflammatory state, and there are higher intra-abdominal pressures in obese patients, which can lead to the formation of venous thrombosis [23]. Studies show that patients who are obese are at a greater risk for thrombosis as well as in patients who are undergoing bariatric surgery, especially for patient with multiple risk factors $[24,25]$. Some risk factors for venous thromboembolism development include obesity, abdominal surgery, smoking, varicose veins, use of oral contraceptives, history of venous thromboembolism, age greater than 60 years old, and documented venous insufficiency [24]. We chose to anticoagulate using IV heparin with bridging to warfarin, consistent with what other studies have done.

\section{Conclusion}

This case study considered a patient who underwent SILSG as surgical management for morbid obesity and subsequently developed SMVT, abdominal compartment syndrome and multiple pulmonary embolisms. While they are rare, these cases carry significant morbidity and mortality, thus requiring immediate attention and treatment. Obese patients who undergo bariatric surgery frequently have other co-morbidities, many of which can complicate a case further. A mesenteric vein thrombosis diagnosis should always be on the differential for abdominal pain, especially if the patient has one or more risk factors. This complication is normally treated with unfractionated or low-molecular-weight heparin.

\section{Competing interests}

The authors declare that they have no competing interests.

\section{References}

1. Wang Y, Beydoun MA, Liang L, Caballero B, Kumanyika SK. Will all Americans become overweight or obese? Estimating the progression and cost of the US obesity epidemic. Obesity (Silver Spring). 2008; 16(10):2323-30.

2. Li JF, Lai DD, Ni B, Sun KX. Comparison of laparoscopic Roux-en-Y gastric bypass with laparoscopic sleeve gastrectomy for morbid obesity or type 2 diabetes mellitus: a meta-analysis of randomized controlled trials. Can J Surg. 2013; 56(6):E158-64.

3. Pineda L, Sarhan M, Ahmed L. Superior mesenteric vein thrombosis after laparoscopic sleeve gastrectomy. Surg Laparosc Endosc Percutan Tech. 2013; 23(4):e162-3.

4. Bellanger DE, Hargroder AG, Greenway FL. Mesenteric venous thrombosis after laparoscopic sleeve gastrectomy. Surg Obes Relat Dis. 2010; 6(1):109-11.

5. Goitein D, Matter I, Raziel A, Keidar A, Hazzan D, Rimon $\mathrm{U}$, et al. Portomesenteric thrombosis following laparoscopic bariatric surgery: incidence, patterns of clinical presentation, and etiology in a bariatric patient population. JAMA Surg. 2013; 148(4):340-6.

6. Morasch MD, Ebaugh JL, Chiou AC, Matsumura JS, Pearce WH, Yao JS. Mesenteric venous thrombosis: a changing clinical entity. J Vasc Surg. 2010; 34(4):680-4.

7. Albeladi B, Bourbao-Tournois C, Huten N. Short- and midterm results between laparoscopic Roux-en-Y gastric bypass and laparoscopic sleeve gastrectomy for the treatment of morbid obesity. J Obes. 2013; 2013:934653.

8. Våge V, Sande VA, Mellgren G, Laukeland C, Behme J, Andersen JR. Changes in obesity-related diseases and biochemical variables after laparoscopic sleeve gastrectomy: a two-year follow-up study. BMC Surg. 2014; $14: 8$.

9. Baltasar A, Serra C, Pérez N, Bou R, Bengochea M, Ferri L. Laparoscopic sleeve gastrectomy: a multi-purpose bariatric operation. Obes Surg. 2005; 15(8):1124-8.

10. Brethauer SA, Hammel JP, Schauer PR. Systematic review of sleeve gastrectomy as staging and primary bariatric procedure. Surg Obes Relat Dis. 2009; 5(4):469-75.

11. Kral JG, Thung SN, Biron S, Hould FS, Lebel S, Marceau $\mathrm{S}$, et al. Effects of surgical treatment of the metabolic syndrome on liver fibrosis and cirrhosis. Surgery. 2004; 135(1):48-58.

12. Wang S, Li P, Sun XF, Ye NY, Xu ZK, Wang D. Comparison between laparoscopic sleeve gastrectomy and laparoscopic adjustable gastric banding for morbid obesity: a meta-analysis. Obes Surg. 2013; 23(7):980-6.

13. Sarkhosh K, Birch DW, Sharma A, Karmali S. Complications associated with laparoscopic sleeve gastrectomy for morbid obesity: a surgeon's guide. Can J Surg. 2013; 56(5):347-52.

14. Melissas J, Koukouraki S, Askoxylakis J, Stathaki M, Daskalakis M, Perisinakis K, et al. Sleeve gastrectomy: a restrictive procedure? Obes Surg. 2007; 17(1):57-62.

15. Csendes A, Burdiles P, Burgos AM, Maluenda F, Diaz JC. Conservative management of anastomotic leaks after 557 open gastric bypasses. Obes Surg. 2005; 15(9):1252-6.

16. Aarts EO, Janssen IM, Berends FJ. The gastric sleeve: losing weight as fast as micronutrients? Obes Surg 2011;21(2):207-11. 17. Mason ME, Jalagani H, Vinik AI. Metabolic complications of bariatric surgery: diagnosis and management issues. Gastroenterol Clin North Am. 2005; 34(1):25-33.

18. Melissas J, Daskalakis M, Koukouraki S, Askoxylakis I, Metaxari M, Dimitriadis E, et al. Sleeve gastrectomy-a "food limiting" operation. Obes Surg. 2008; 18(10):1251-6.

19. Birkmeyer NJ, Finks JF, Carlin AM, Chengelis DL, Krause KR, Hawasli AA, et al. Comparative effectiveness of unfractionated and low-molecular-weight heparin for prevention of venous thromboembolism following bariatric surgery. Arch Surg .2012; 147(11): 994-8.

20. Palmer AJ1, Schramm W, Kirchhof B, Bergemann R. Low molecular weight heparin and unfractionated heparin for prevention of thrombo-embolism in general surgery: a metaanalysis of randomised clinical trials. Haemostasis. 1997; 27(2):65-74.

21. Swartz DE, Felix EL. Acute mesenteric venous thrombosis 
following laparoscopic Roux-en-Y gastric bypass. JSLS. 2004; 8(2):165-9.

22. Frederiksen SG, Hedenbro JL, Norgren L. Enoxaparin effect depends on body-weight and current doses may be inadequate in obese patients. Br J Surg. 2003; 90(5): 547-8.

23. Gandhi K, Singh P, Sharma M, Desai H, Nelson J, Kaul A. Mesenteric vein thrombosis after laproscopic gastric sleeve procedure. J Thromb Thrombolysis. 2010; 30(2):179-83.

24. Hamad GG, Choban PS. Enoxaparin for thromboprophylaxis in morbidly obese patients undergoing bariatric surgery: findings of the prophylaxis against VTE outcomes in bariatric surgery patients receiving enoxaparin (PROBE) study. Obes Surg. 2005; 15(10):1368-74.

25. Rocha AT, de Vasconcellos AG, da Luz Neto ER, Araújo DM, Alves ES, Lopes AA. Risk of venous thromboembolism and efficacy of thromboprophylaxis in hospitalized obese medical patients and in obese patients undergoing bariatric surgery. Obes Surg. 2006; 16(12):1645-55. 